\title{
Hunting for Gamma Ray Bursts with Pi of the Sky telescopes in Chile and Spain
}

M. Siudek ${ }^{* 1}$, T. Batsch ${ }^{2}$, H. Czyrkowski ${ }^{3}$, M. Cwiok $^{3}$, R. Dabrowski ${ }^{3}$, G. Kasprowicz $^{4}$, A. Majcher ${ }^{2}$, A. Majczyna ${ }^{2}$, K. Malek ${ }^{1,5}$, L. Mankiewicz ${ }^{1}$, K. Nawrocki $^{2}$, R. Opiela ${ }^{1}$, L. W. Piotrowski ${ }^{3}$, M. Sokolowski ${ }^{2}$, R. Wawrzaszek ${ }^{6}$, G. Wrochna ${ }^{2}$, M. Zaremba $^{3}$, A. F. Żarnecki ${ }^{3}$

${ }^{1}$ Centre for Theoretical Physics of the Polish Academy of Sciences, Al. Lotnikow 32/46, 02-668 Warsaw, Poland

${ }^{2}$ National Centre for Nuclear Research, Hoza 69, 00-681 Warsaw, Poland

${ }^{3}$ Faculty of Physics, University of Warsaw, Hoza 69, 00-681 Warsaw, Poland

${ }^{4}$ Institute of Electronic Systems, Warsaw University of Technology, Nowowiejska 15/19, 00-665 Warsaw, Poland

${ }^{5}$ Institute for Advanced Research, Nagoya University, Furo-cho, Chikusa-ku, 464-8601 Nagoya, Japan

${ }^{6}$ Space Research Center of the Polish Academy of Sciences, Bartycka 18A, 00-716 Warsaw,

Poland

E-mail: gsiudekecft.edu.pl

Pi of the Sky is a system of wide field-of-view robotic telescopes which search for short timescale astrophysical phenomena, especially for prompt optical GRB emission. The system is designed for autonomous operation, follows the predefined observing strategy and adopts it to the actual conditions. Simultaneous observations from locations in Chile and Spain allow a systematic search for optical transients of cosmological origin. Accurate analysis of data arising from a wide-field system like Pi of the Sky is a real challenge because of a number of factors that can influence the measurements. We have developed a set of dedicated algorithms which remove poor quality measurements, improve photometric accuracy and allow us to reach uncertainties as low as $0.015-0.02 \mathrm{mag}$.

Gamma-Ray Bursts 2012 Conference-GRB2012,

May 07-11, 2012

Munich, Germany

\footnotetext{
${ }^{*}$ Speaker.
} 


\section{Introduction}

The "Pi of the Sky" [1] is a system of wide field of view robotic telescope designed for efficient search for astrophysical phenomena varying on scales from seconds to months, especially for prompt optical counterparts of Gamma Ray Bursts (GRBs). The design of the apparatus allows to monitor a large fraction of the sky with a range of $12^{m}-13^{m}$ and time resolution of the order of $1-10$ seconds. In order to ensure that all project requirements are met with full control over the detector design and construction, "Pi of the Sky" detectors are equipped with custom designed CCD cameras, build by project members. Each camera is equipped with Canon lenses $\mathrm{f}=85 \mathrm{~mm}$, $\mathrm{f} / \mathrm{d}=1.2$ and covers $20^{\circ} \times 20^{\circ}$ of the sky.

The main goal of the "Pi of the Sky" project is to search for and observe prompt optical counterparts of GRBs during or even before gamma-ray emission. To achieve this purpose "Pi of the Sky" selected an approach which assumes continuous observation of a large part of the sky to increase the possibility of catching a GRB. Therefore, it is necessary to develop advanced and fully automatic software for real-time data analysis and identification of flashes.

The full "Pi of the Sky" system, which is under construction now, will be capable of continuous observation of about 1.5 steradians, which is roughly corresponding to the field of view of the BAT instrument on board the Swift satellite [2]. We have designed a system consisting of 2 sites separated by a distance of about $100 \mathrm{~km}$ allowing to reject flashes from satellites and other nearEarth objects by parallax. Each site will consist of 12 custom-designed CCD cameras placed on specially designed equatorial mounts (4 cameras per mount, see Fig. 1). In October 2010 the first unit of the new Pi of the Sky detector system was successfully installed in the INTA El Arenosillo Test Centre in Spain. The first site of the ultimate system should be fully operational this year.

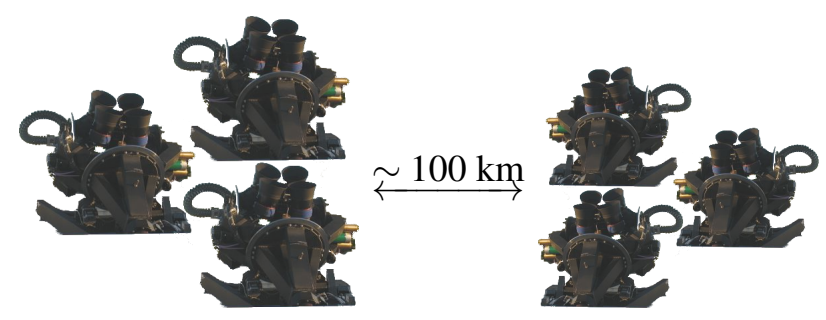

Figure 1: A scheme of the final system.

\subsection{Observational strategy - continuous observation of large part of the sky}

Observations of optical counterparts of GRBs during or even before the gamma-ray emission are crucial for understanding the nature of GRBs. The standard approach, which relies on waiting for an alert distributed by the GCN network (The Gamma Ray Burst Coordinates Network) [3] and subsequently moving the telescopes to the target as fast as possible, does not allow us to detect an outburst at the moment of or before the GRB explosion. Thus, the "Pi of the Sky" strategy is based on continuous observation of a large fraction of the sky, which increases the chances that a GRB will occur in the observed area. Following the field of view of the Swift satellite, with the full "Pi of the Sky" system, will allow to eliminate a delay of the observation due to telescope 
re-pointing to the coordinates from GCN. Dead time, which arises from the decision process and signal propagation from the satellite to the GCN and from the GCN to the ground instruments is eliminated as well.

The search for GRB requires very fast data processing and identification of events in real-time. On the other hand, the search for transients and the analysis of variable star are based on precise photometry, which requires detailed image analysis. To fulfill both requirements we developed two different sets of algorithms: for on-line and off-line data processing. Off-line analysis is to identify all objects in an image, and to add their measurements to the database. The on-line algorithm searches for flashes in real-time by comparing a new image with the stack of recently taken frames. Any observed difference is considered as possible candidate event. All events are processed through a multilevel triggering system similar to those known from high-energy physics experiments.

The observations of the famous "naked-eye" GRB080318B [4] have confirmed the usefulness of "Pi of the Sky" strategy. Wide-field telescopes performing continuous observations of large part of the sky are capable of detecting GRBs at the moment or even before explosion. GRB080318B was recognized by the "Pi of the Sky" self-triggering system independently from the alert received from the GCN.

\subsection{The "Pi of the Sky" prototype}

Before constructing the final version, tests of hardware and software were performed with a prototype consisting of 2 custom-designed cameras placed on an equatorial mount. The detector is fully autonomous and operates without any human supervision, although remote control via Internet is possible as well. Cameras work in coincidence and observe the same field of view with a time resolution of $10 \mathrm{~s}$. The limiting magnitude for a single frame is $12^{m}$ and rises to $13.5^{m}$ for a frame stacked from 20 exposures. Till 2009 all observations were made in white light and no filter was used, except for an IR-cut filter in order to minimize the sky background. Since May 2009 we have had a Bessel-Johnson R-band filter installed on one of the cameras in order to facilitate absolute calibration of the measurements. The prototype had been working at Las Campanas Observatory in Chile since June 2004 till the end of 2009. In March 2011 the detector was moved to a new site in San Pedro de Atacama, approximately $750 \mathrm{~km}$ north from LCO (still in Chile) and about 2400 meters above sea level (see Fig. 2).
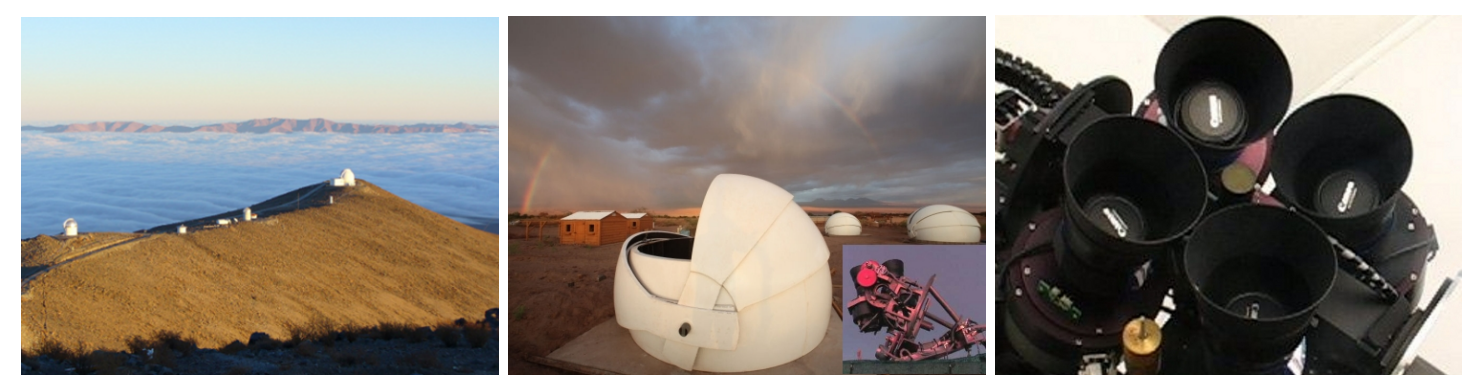

Figure 2: The "Pi of the Sky" prototype was moved from Las Campanas Observatory (left) to San Pedro de Atacama (middle) in March 2011. The final detector operates in two modes: side-by-side (right) or common target. 


\subsection{New detector unit in Spain}

The final detector consists of 4 custom-designed CCD cameras, which are improved versions of the cameras developed for the prototype. The cameras can operate in two operational modes thanks to specially designed equatorial mount. The mechanism for deflecting cameras enable to point all cameras at the same object (common-target mode, DEEP, common field of view $20^{\circ} \times 20^{\circ}$ ) or cameras are deflected by $15^{\circ}$ along the diagonal of the CCD chip, covering adjacent field (sideby-side, WIDE, total coverage $40^{\circ} \times 40^{\circ}$ ) (see Fig. 2). Due to numerous improvements, the new design of the telescope mount provides much better pointing accuracy and a shorter reaction time than the prototype. New detector unit has been successfully operated in the INTA El Arenosillo test centre in Mazagón near Huelva, Spain, on the coast of the Atlantic Ocean from October 2010.

\section{The "Pi of the Sky" data}

All data gathered by the "Pi of the Sky" prototype located in Chile are stored in publicly accessible databases ${ }^{1}$. Currently three databases are available:

1. May 2006 - April 2009 containing over 2 billion measurements for almost 17 million objects

2. July 2004 - June 2005 containing almost 800 million measurements for about 4.5 million objects

3. May 2006 - November 2007 (subset of the first database) containing over 1000 million measurements for almost 11 million objects

Access to databases is through a user-friendly web interface, which allows to select objects according to their type, magnitude, coordinates etc, displaying their light curves and other properties. It is also possible to download large packets of light curves of multiple stars. Moreover we have developed a system of dedicated filters and color calibration algorithms to improve quality of our data.

\subsection{Towards better photometry accuracy}

We have develop a series of quality filter cuts to remove measurements (or whole frames) affected by detector imperfections or observing conditions. Measurements that are placed near the border of the frame, or that are affected by hot pixels, bright background caused by open shutter or moon halo, or by planet or planetoid passage, can be easily removed from the light curves of stars. Photometry accuracy obtained after excluding affected measurements can be significantly improved. For stars with range $7-10^{m}$, average photometric uncertainty of about $0.018-0.024^{m}$ has been achieved (see fig. 3).

We also managed to improve photometry uncertainty by developing approximate color calibration algorithm. When we analyzed the variable star BG Ind [5], it turned out that the "Pi of the Sky" detector response is correlated with the stellar spectral type (performing observations without any filter, except IR-UV-cut ${ }^{1}$ filter, the detectors have a relatively wide spectral sensitivity). The average magnitude measured by "Pi of the Sky" $\left(M_{P I}\right)$ is shifted with respect to the catalog magnitude in $\mathrm{V}$ band by an offset depending on the spectral type given by $\mathrm{B}-\mathrm{V}$ or $\mathrm{J}-\mathrm{K}$ (see fig. 4).

\footnotetext{
${ }^{1} \mathrm{Pi}$ of the Sky Home Page: http ://grb.fuw.edu.pl/pi/

${ }^{1}$ From May 2009 we have a standard R filter installed on one of cameras
} 

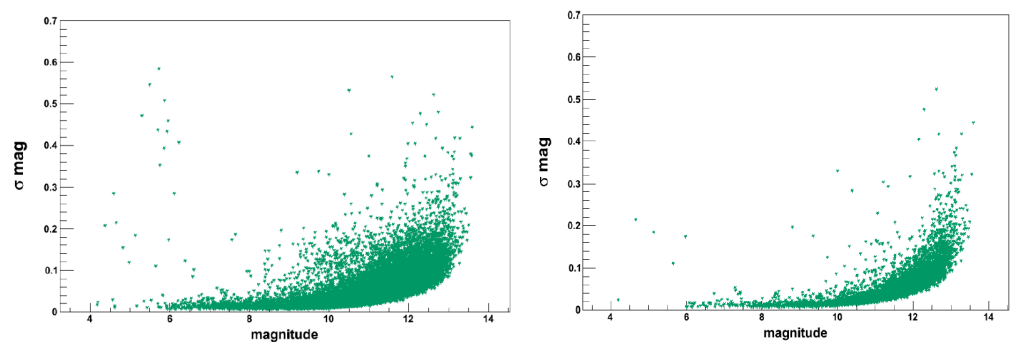

Figure 3: Precision of star magnitude measurements from standard photometry for 200s exposures (20 coadded frames) from the "Pi of the Sky" prototype in the Las Campanas Observatory in Chile. Large dispersion (left) is mainly caused by false measurements. After the application of quality cuts (right) photometry accuracy improves significantly.

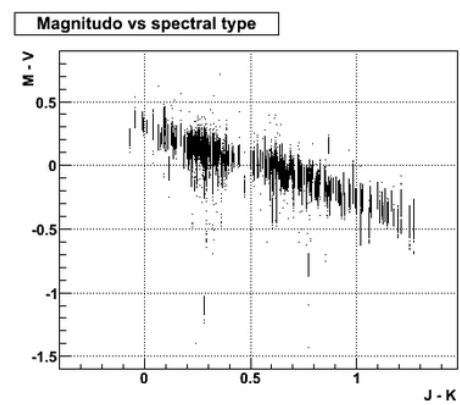

Figure 4: Average difference between the "Pi of the Sky" magnitude and catalog V magnitude for reference stars, as a function of the spectral type given by $\mathrm{J}-\mathrm{K}$.

The approximation of this dependence with a linear function enables the measurement of each star to be corrected, so that measured magnitude $\left(M_{\text {coor }}\right)$ is equal to catalog V magnitude independently of the spectral type. Significant improvement of the measurement precision is also achieved when the photometric correction is not calculated as a simple average over all selected reference stars, but when a quadratic dependence of the correction on the reference star position in the sky is fitted for each frame. The distribution of $\chi^{2}$ can be used to select measurements with the most precise photometry. Applying the new algorithm to the light curve of BG Ind improve the photometric quality, and a brightness uncertainty of the order of $0.013^{m}$ was obtained (see fig. 5).
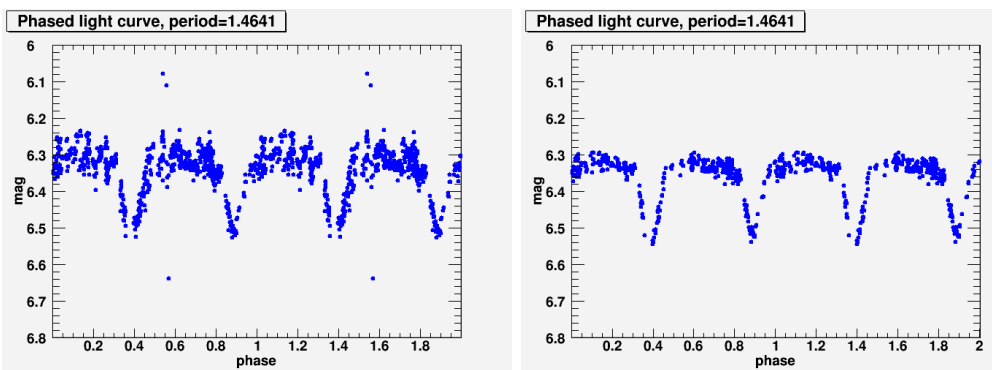

Figure 5: Uncorrected light curve for BG Ind variable (left) and after spectral corrections (right). 


\section{Conclusions}

The "Pi of the Sky" instruments operate in a fully autonomous mode, practically without any human supervision, and search for short-timescales astrophysical phenomena, especially for prompt optical counterparts of GRBs. The ultimate system will be able to perform continuous observations of the field of view of Swift satellite, which will allow to detect GRBs at the moment or even before the explosion. The observations of the famous "naked-eye" GRB080318B with the prototype located in Las Campanas Observatory in Chile, have confirmed the power of the observing strategy of "Pi of the Sky".

The first site of the ultimate system should be operational this year. The first unit of the new "Pi of the Sky" detector system has been successfully performing observations in the INTA El Arenosillo Test Centre in Spain since October 2010. The prototype, after moving from Las Campanas Observatory to San Pedro de Atacama Observatory in March 2011, is effectively collecting new data as well.

During the period 2006-2009 the prototype has gathered over 2 billion measurements for almost 17 million objects. All measurements acquired by "Pi of the Sky" are publicly accessible through a user-friendly web interface on the Pi of the Sky Home Page. Effort on improving data quality is still ongoing. We have developed a system of dedicated filters to remove measurements from star light curve measurements, which could be affected by different factors due to detector imperfections or weather conditions. The measurement quality can be improved by an approximate color calibration algorithm based on the spectral type of reference stars and an uncertainty of the order of $0.013^{m}$ can be obtained.

\section{Acknowledgements}

We are very grateful to G. Pojmanski for access to the ASAS dome and sharing his experience with us. We would like to thank the staff of the Las Campanas Observatory, San Pedro de Atacama Observatory and the INTA El Arenosillo test centre in Mazagón near Huelva for their help during the installation and maintenance of our detector. This work has been financed by the Polish Ministry of Science and Higher Education in 2009-2011 as a research project.

\section{References}

[1] A. Burd, M. Cwiok, H. Czyrkowski, et al., Pi of the Sky - all-sky, real-time search for fast optical transients, New A 10 (2005) 409 [astro-ph / 0411456 ].

[2] N. Gehrels, et al., The Swift Gamma-Ray Burst Mission, ApJ 611 (2004) 1005.

[3] S. D. Barthelmy, et al., The GRB coordinates network (GCN): A status report, AIPC 428 (1997) 99.

[4] J. L. Racusin, S. V. Karpov, M. Sokolowski, et al., Broadband observations of the naked-eye $\gamma$-ray burst GRB080319B, Nature 455 (2008) 183 [astro-ph / 0805 . 1557].

[5] M. Rozyczka, K. Malek, et al., Absolute properties of BG Ind - a bright F3 system just leaving the main sequence, MNRAS 414 (2011) 2479 [astro-ph/ 1010.1355 ]. 